\title{
Transferencia de Calor y Materia durante el Proceso de Freído de Alimentos: Tilapia (Oreochromis niloticus) y Fruta de Pan (Artocarpus communis)
}

\author{
Diego F. Tirado*, Diofanor Acevedo y Piedad M. Montero \\ Universidad de Cartagena, Facultad de Ingeniería, Programa de ingeniería de Alimentos, Avenida el \\ Consulado, Calle 30 No. 48-152. Cartagena, Bolívar-Colombia (e-mail: dtiradoa@unicartagena.edu.co) \\ * autor a quien debe ser dirigida la correspondencia
}

Recibido Abr. 21, 2014; Aceptado Jun. 30, 2014; Versión final recibida Sep. 22, 2014

\begin{abstract}
Resumen
En este estudio se determinaron los coeficientes de transferencia de masa, difusividad y los coeficientes convectivos de transferencia de calor durante el freído por inmersión de láminas de tilapia y fruta de pan. La determinación se realizó en aceite de palma a 130,150 y $170^{\circ} \mathrm{C}$ para la tilapia y en aceite de soya a 150 , 170 y $190^{\circ} \mathrm{C}$ para la fruta de pan. Las propiedades termofísicas se midieron en función de la temperatura y composición del alimento. Los coeficientes convectivos fueron determinados a partir de gráficas de relaciones adimensionales tiempo vs. Temperatura. Los coeficientes aumentaron al aumentar la temperatura del aceite para la fruta de pan y disminuyeron al aumentar la temperatura del aceite para la tilapia. Los coeficientes de transferencia de masa fueron determinados a partir de las graficas adimensionales de concentración de humedad contra el tiempo y estos aumentaron con la temperatura de fritura.
\end{abstract}

Palabras clave: Oreochromis niloticus, Artocarpus communis, freído por inmersión, transferencia de masa, transferencia de calor

\section{Heat and Mass Transfer during Frying of Food: Tilapia (Oreochromis niloticus) and Pan fruit (Artocarpus communis)}

\begin{abstract}
In this study, the mass transfer coefficients, diffusivity and convective heat transfer coefficients during frying dip sheet and breadfruit tilapia were determined. The determination was made in palm oil at 130,150 and $170^{\circ} \mathrm{C}$ for tilapia and soybean oil at 150,170 and $190^{\circ} \mathrm{C}$ for breadfruit. The thermophysical properties were measured as a function of temperature and composition of the food. The convective coefficients were determined from plots of dimensionless relationships time vs. temperature. The coefficients these increased with increasing oil temperature for breadfruit and decreased with increasing oil temperature for tilapia. The mass transfer coefficients were determined from the graphs of dimensionless moisture concentration against time and these increased with frying temperature.
\end{abstract}

Keywords: Oreochromis niloticus, Artocarpus communis, immersion frying, mass transfer, heat transfer 


\section{INTRODUCCIÓN}

A nivel mundial las tilapias (Oreochromis niloticus) son el tercer grupo de peces de mayor importancia, después de la carpa y el salmón, y su cultivo también es una de las actividades de mayor crecimiento (Tirado et al., 2013). Por otro lado el árbol del pan (Artocarpus altilis) es una especie perteneciente al género de los Artocarpus, dentro de la tribu de las Artocarpeae, de la familia de las Moraceae con cientos de variedades de árboles distribuidas desde el sudeste asiático hasta la Polinesia, pasando por Oceanía. La especie más conocida es la Artocarpus altilis, o árbol del pan propiamente dicho. La fruta del pan (Artocarpus communis) es el fruto tropical que se obtiene del árbol del pan. Tiene ese nombre, debido a que su pulpa tiene un aspecto similar al del pan. Es la parte comestible del árbol del pan, recurso natural valioso debido a su alto contenido en nutrientes: $84,2 \%$ de carbohidratos, proteínas de $4,1 \%$, fibra de 3,5\% y $2,9 \%$ de minerales en peso seco (Hsu et al., 2011).

Freír por inmersión, puede ser definido como el proceso de cocción de alimentos por inmersión en un aceite o grasa comestible que está a una temperatura superior al punto de ebullición del agua, por lo general 150 a $200{ }^{\circ} \mathrm{C}$ (Lalam et al., 2013; Tirado et al., 2012; Mir-Bel et al., 2012). En esencia, es un proceso de deshidratación rápida (Wu et al., 2013b), en el que contribuyen factores como la temperatura, tiempo de calentamiento, tipo de aceite, tamaño y naturaleza del producto (Mir-Bel et al., 2012). El gran empleo del freído en hogares e industria se debe principalmente a que los alimentos, tras este proceso, adquieren unas características únicas de aroma y textura que no es posible conseguir con otras técnicas de procesado de alimentos (Ahmad et al., 2013; Aladedunye y Przybylski, 2013), siendo así un campo de gran interés para los investigadores y científicos de ingeniería, así como de diseñadores, desarrolladores y fabricantes (Wu et al., 2013a).

Además de los cambios organolépticos que provoca la fritura, un efecto adicional es la conservación del alimento, como resultado de la destrucción de los microorganismos e inactivación de enzimas por efecto del calor y de la reducción de la actividad de agua (Tirado et al., 2012). Sin embargo, los estudios en ingeniería son escasos, y los snacks como las patatas fritas, son los productos más estudiados (Sosa-Morales et al., 2006). A través del uso de modelos matemáticos, es posible el diseño de los procesos de fritura (Nasiri et al., 2011). La fritura implica transferencia simultánea de calor y masa, por lo que es necesario el conocimiento de los parámetros de proceso críticos para los procesos de modelado (Yildiz et al., 2007).

Según Alvis et al., (2009a) el modelado y los cálculos de los sistemas de freído de alimentos, para el diseño de equipos y procesos de elaboración, tienen que ser evaluados y utilizarse con cuidado para comprobar la calidad de los alimentos que consumimos. A pesar de ser tan importante la determinación de los coeficientes de transferencia de calor y masa, los métodos reportados en la literatura para medir dicho coeficiente presentan algunas limitaciones (Tirado et al., 2012; Alvis et al., 2009; Moreira et al., 1999) tales como: a) Faltan técnicas estándar de medida, b) Pocas veces se toma en consideración la transferencia de masa, c) Escaso conocimiento del coeficiente de transferencia de calor (en función de la posición del material), d) No existe uniformidad en la estimación y reporte de errores de medida, e) Geometría del producto. El último inconveniente se refiere a que se hace tedioso acoplar modelos matemáticos a formas de productos no convencionales, y además, debido a que el producto presenta deshidratación durante el proceso, este pierde masa y su forma, con lo cual se presenta una variación en su espesor. Para poder acabar con este tipo de problemas se ensaya con productos de geometrías convencionales que faciliten cálculos: cilindros, esferas y láminas. Esta última fue la geometría utilizada en este trabajo. Y ya que como se mencionó anteriormente el espesor de estas láminas varían por la pérdida de masa del material y diversas reacciones de las macromoléculas del alimento, se decide seleccionar un espesor promedio, ya que así se pueden modelar más sencillamente los modelos matemáticos como el usado en esta y otras investigaciones que han considerado el espesor de la lámina constante, esto para hacer más fácil el estudio del proceso, ya que uno de las principales inconvenientes en estos estudios es la geometría del sistema (Tirado et al., 2012; Alvis et al., 2009; Moreira et al., 1999).

En las últimas décadas la transferencia de calor y masa durante la fritura de algunos alimentos fueron atendidos por algunos investigadores. Alvis et al., (2009b) estudiaron la transferencia de calor y masa durante el freído de trozos de ñame. Sosa-Morales et al., (2006) analizaron la pérdida de agua y transferencia de calor en la fritura de tiras de cerdo; y Dincer y Yildiz (1996) propusieron un modelo de estado no estacionario para la transferencia de calor y masa en los embutidos durante la fritura. No se encontraron investigaciones concernientes al estudio de la cinética de transferencia de calor y masa durante la fritura de tilapia o fruta del pan, por lo que el presente trabajo tuvo como objetivo determinar los coeficientes convectivos de transferencia de calor y los coeficientes de transferencia de masa durante el proceso de freído de tilapia (O. niloticus) y fruta de pan (Artocarpus communis) a diferentes temperaturas a partir de la solución analítica de las ecuaciones diferenciales de difusión de masa y conducción de calor para una placa infinita de espesor $2 \mathrm{~L}$, y su importancia frente al uso de el análisis de parámetros concentrados utilizado por algunos autores. 


\section{MATERIALES Y MÉTODOS}

\section{Coeficientes de transferencia de masa}

Para la determinación de coeficientes de transferencia de masa se utilizaron filetes de tilapia (O. niloticus) en forma de placa plana de $0,02^{*} 0,02^{*} 0,02 \mathrm{~m}$, y trozos de fruta de pan ( $A$. communis) en forma de paralelepípedo de $0,008^{*} 0,008^{*} 0,04 \mathrm{~m}$. En el freído se empleó aceite de palma para la tilapia y aceite de soya para la fruta de pan como medio de fritura y una freidora eléctrica en acero inoxidable de 6 litros de capacidad con temperatura controlada por termostato. Las muestras se colocaron en una cesta de alambre y luego fueron sumergidas para los tiempos de $0,60,120,180,240$, y 300 s a 130, 150 y $170^{\circ} \mathrm{C}$ para las láminas de tilapia y a 150,170 y $190^{\circ} \mathrm{C}$ a tiempos de $0,5,10,20,30,35,40,45,50,60,70,80,100,120$, 130,140 s para los trozos de fruta de pan. Estas diferencias en tiempos y temperaturas se deben a las condiciones normales de freído de ambos alimentos. Dos muestras se fritaron en cada lote para reducir las fluctuaciones de temperatura durante la fritura. El aceite de fritura fue remplazado después de cada lote. Se sacaron muestras y se colocaron en papel filtro para remover el aceite superficial e inmediatamente se les determinó la humedad a $105 \stackrel{\circ}{\circ} \mathrm{C}$ hasta peso constante (A.O.A.C., 2003). Los datos de contenido de humedad de las láminas en función del tiempo se utilizaron posteriormente en un modelo matemático para la determinación del coeficiente de transferencia de masa. Se utilizó para ello la ecuación de concentración en función del tiempo y la posición, para una lámina infinita de espesor $2 \mathrm{~L}$, que se obtiene resolviendo la ecuación diferencial de difusión en estado transitorio mostrada en la Ecuación (1), con las condiciones límites e inicial expresados en la Ecuación (2) tal como lo hizo Yildiz et al., (2007) en fritura de papas. Al igual que estos autores se considera el espesor de la lámina como un valor promedio, para estimar la geometría de lámina del producto constante durante el proceso y así hacer válido el modelo matemático utilizado.

$\frac{\partial^{2} C}{\partial x^{2}}=\frac{1}{D} \frac{\partial C}{\partial t}, 0 \leq x \leq L$ for $t>0$

$\frac{\partial C}{\partial x}=0 x=0 ;-D \frac{\partial C}{\partial x}=k_{C}\left(C-C_{\infty}\right)$ en $x=L ; C=C_{i}$ cuando $t=0$

La siguiente solución como serie infinita muestra la concentración de la humedad localizada en cualquier punto dentro de la lámina como una función del tiempo.

$\left(\frac{C(x, t)-C_{\infty}}{C_{i}-C_{\infty}}\right)=\sum_{n=0}^{n=\infty} \frac{2 \operatorname{Sen} \delta_{n}}{\delta_{n}+\left(\operatorname{Sen} \delta_{n}\right)\left(\operatorname{Cos} \delta_{n}\right)} \operatorname{Cos}\left(\delta_{n} \frac{x}{L}\right) e^{-\delta_{n}^{2} \frac{D t}{L^{2}}}$

La forma reducida de la Ecuación (3) puede ser usada para obtener la solución para láminas en forma de paralelepípedo (finito en dos dimensiones), haciendo uso de la regla de superposición, según la cual, la solución para la transferencia de masa de una lámina finita en 2 dimensiones se encuentra multiplicando la solución para 2 láminas infinitas, Ecuación (4), desarrollada por Crank (1975).

$\left(\frac{C(x, y, t)-C_{\infty}}{C_{i}-C_{\infty}}\right)_{\text {Lámina finita }}=\left(\frac{C(x, t)-C_{\infty}}{C_{i}-C_{\infty}}\right)_{\text {Lámina infinita } 2 L}\left(\frac{C(y, t)-C_{\infty}}{C_{i}-C_{\infty}}\right)_{\text {Lámina infinita } 2 L}$

Donde $\mathrm{C}(\mathrm{x}, \mathrm{y}, \mathrm{t})$ es la concentración de humedad en cualquier punto y en cualquier tiempo, ( $\mathrm{kg} / \mathrm{kg}$ sólidos). Remplazando en (4) para placas infinitas de espesor finito en $x$ e $y$, se obtienen las Ecuaciones (5) y (6).

$$
\begin{aligned}
& \left(\frac{C(x, t)-C_{\infty}}{C_{i}-C_{\infty}}\right)=\frac{2 \operatorname{Sen} \delta_{n}}{\delta_{n}+\left(\operatorname{Sen} \delta_{n}\right)\left(\operatorname{Cos} \delta_{n}\right)} \operatorname{Cos}\left(\delta_{n} \frac{x}{L}\right) e^{-\delta_{m}^{2} \frac{D t}{L^{2}}} \\
& \left(\frac{C(y, t)-C_{\infty}}{C_{i}-C_{\infty}}\right)=\frac{2 \operatorname{Sen} \delta_{n}}{\delta_{n}+\left(\operatorname{Sen} \delta_{n}\right)\left(\operatorname{Cos} \delta_{n}\right)} \operatorname{Cos}\left(\delta_{n} \frac{y}{L}\right) e^{-\delta_{m}^{2} \frac{D t}{L^{2}}}
\end{aligned}
$$

El producto de la Ecuación (5) por la Ecuación (6) da como resultado la Ecuación (7):

$$
\left(\frac{C(x, t)-C_{\infty}}{C_{i}-C_{\infty}}\right)\left(\frac{C(y, t)-C_{\infty}}{C_{i}-C_{\infty}}\right)=E e^{\left(-\delta_{n}^{2} \frac{D_{a} t}{L^{2}}\right)}
$$

Donde: 
$E=\frac{2 \operatorname{Sen} \delta_{n}}{\delta_{n}+\left(\operatorname{Sen} \delta_{n}\right)\left(\operatorname{Cos} \delta_{n}\right)}$

Linealizando la Ecuación (7) nos queda la Ecuación (9):

$\ln \left(\frac{C(x, y, t)-C_{\infty}}{C_{i}-C_{\infty}}\right)=2 \ln E-2 \delta_{n}^{2} \frac{D_{a} t}{L^{2}}$

Graficando $\operatorname{Ln}\left(\frac{C(x, y, t)-C_{\infty}}{C_{i}-C_{\infty}}\right)$ vs t, desde el intercepto de la gráfica fue calculada la primera raíz de la ecuación característica $\left(\delta_{\mathrm{n}}\right)$. Después se determinó el coeficiente de difusión, $\mathrm{D}_{\mathrm{a}}\left(\mathrm{m}^{2} / \mathrm{s}\right)$ desde la pendiente de la sección lineal de esta grafica que se iguala a $-2 \delta_{n}^{2} \frac{D_{a}}{L^{2}}$. Una vez hallado $\delta_{\mathrm{n}}$ se determina $\mathrm{Bi}_{\mathrm{m}}$ y posteriormente $\mathrm{k}_{\mathrm{c}}$.

$B i_{m}=\delta_{n} \operatorname{Tan} \delta_{n}$

$B i_{m}=\frac{k_{c} L}{D_{a}}$

\section{Coeficientes convectivos de transferencia de calor}

Para la determinación del coeficiente convectivo de transferencia de calor se utilizaron filetes de tilapia en forma de placa plana de $0,05^{\star} 0,025^{\star} 0,015 \mathrm{~m}$ y trozos de fruta de pan de $0,008^{\star} 0,008^{\star} 0,04 \mathrm{~m}$. Las condiciones de proceso fueron temperaturas de fritura de 130,150 y $170^{\circ} \mathrm{C}$ para láminas de tilapia y 150 , 170 y $190^{\circ} \mathrm{C}$ para los trozos fruta de pan. Los tiempos de freído fueron entre 1 a $420 \mathrm{~s}$ para la tilapia y de 5 a $180 \mathrm{~s}$, con variaciones de $5 \mathrm{~s}$ para la fruta de pan. Para medir la variación de temperatura de las láminas se insertaron 2 termopares tipo $\mathrm{J}$ de $0,00025 \mathrm{~m}$ de diámetro por los extremos de la placa en sentido longitudinal hasta que las puntas de los mismos estuvieron cerca del centro geométrico y suficientemente lejos de los extremos; otro termopar de las mismas características se usó para medir la temperatura del aceite. Las temperaturas se registraron cada segundo empleando un sistema de adquisición de datos (INTECH Micro 2100-A16 Rev 1.3) acoplado a un computador personal. Las propiedades termofísicas de ambos alimentos se determinaron usando un programa computacional denominado CTCIA (Coeficientes de Transferencia de Calor en Ingeniería de Alimentos) de Tirado et al., (2014).

El coeficiente de transferencia de calor se determinó a partir de la solución analítica de la ecuación diferencial de conducción de calor en una dimensión en coordenadas cartesianas para una placa infinita de espesor $2 \mathrm{~L}$ en forma análoga a la transferencia de masa tal como lo utilizó Alvis et al., (2009) y Yildiz et al., (2007). La resolución y linealización queda expresada como se indica en la Ecuación (12). Aquí también se considera el espesor de lámina constante durante el proceso.

$\ln \left(\frac{T(x, y, t)-T_{\infty}}{T_{i}-T_{\infty}}\right)=\ln A-2 \delta_{n}^{2} \frac{\alpha t}{L^{2}}$

Donde:

$A=\frac{2 \operatorname{Sen} \delta_{n}}{\delta_{n}+\left(\operatorname{Sen} \delta_{n}\right)\left(\operatorname{Cos} \delta_{n}\right)} \operatorname{Cos}\left(\delta_{n} \frac{x}{L}\right) \operatorname{Cos}\left(\delta_{n} \frac{y}{L}\right)$

Graficando $\ln \left(\frac{T(x, y, t)-T_{\infty}}{T_{i}-T_{\infty}}\right)$ vs t, la pendiente de la sección lineal de esta gráfica es igual a $\delta_{n}^{2} \frac{\alpha}{L^{2}}$. Conocidos $\alpha$ (Difusividad térmica en $\mathrm{m}^{2} / \mathrm{s}$ ) y $\mathrm{L}$ (Mitad del espesor en $\mathrm{m}$ ) se determina $\delta_{n}$. Una vez hallado $\delta_{n}$, se determina Bi y posteriormente $h$, así:

$$
\begin{aligned}
& B i=\delta_{n} \operatorname{Tan}_{n} \\
& B i=\frac{h L}{k}
\end{aligned}
$$




\section{RESULTADOS Y DISCUSIÓN}

Se presentan los resultados de los coeficientes de transferencia de masa y coeficientes de transferencia de calor. Se presentan también los datos de pérdida de humedad durante el freido y los diagramas de la relación adimensional de concentración de humedad vs. Tiempo.

Coeficientes de transferencia de masa

Tabla1: Pérdida de humedad durante el freído de láminas de tilapia

\begin{tabular}{|c|c|c|c|}
\hline \multirow{2}{*}{ Tiempo (s) } & \multicolumn{3}{|c|}{ \% Humedad } \\
\cline { 2 - 4 } & $130^{\circ} \mathrm{C}$ & $150^{\circ} \mathrm{C}$ & $170^{\circ} \mathrm{C}$ \\
\hline 0 & 64,60 & 64,60 & 64,60 \\
\hline 60 & 63,78 & 63,67 & 60,07 \\
\hline 120 & 63,51 & 60,30 & 57,85 \\
\hline 180 & 63,17 & 58,52 & 50,01 \\
\hline 240 & 59,15 & 55,92 & 45,36 \\
\hline 300 & 53,11 & 51,88 & 36,22 \\
\hline
\end{tabular}

Tabla 2: Pérdida de humedad durante el freído de fruta de pan

\begin{tabular}{|c|c|c|c|}
\hline Tiempo $(\mathrm{s})$ & $150^{\circ} \mathrm{C}$ & $150^{\circ} \mathrm{C}$ & $190^{\circ} \mathrm{C}$ \\
\hline 0 & 77 & 77 & 77 \\
\hline 5 & 76,76 & 76,84 & 76,92 \\
\hline 10 & 70,44 & 72,58 & 73,61 \\
\hline 20 & 63,04 & 63,73 & 64,63 \\
\hline 30 & 54,26 & 58,19 & 59,96 \\
\hline 35 & 51,61 & 52,65 & 54,26 \\
\hline 40 & 50,08 & 51,10 & 52,13 \\
\hline 45 & 42,68 & 46,70 & 51,61 \\
\hline 50 & 35,29 & 37,47 & 42,68 \\
\hline 60 & 29,77 & 34,59 & 42,25 \\
\hline 70 & 28,89 & 33,57 & 41,83 \\
\hline 80 & 25,63 & 33,24 & 40,19 \\
\hline 100 & 23,19 & 33,24 & 40,15 \\
\hline 120 & 22,96 & 33,20 & 40,11 \\
\hline 130 & 22,96 & 33,17 & 40,11 \\
\hline 140 & 22,96 & 70,78 & 40,11 \\
\hline
\end{tabular}

Los diagramas de la relación adimensional de concentración de humedad vs. tiempo obtenidos para la tilapia y la fruta de pan son presentados en las Figura 1 y 2 respectivamente. Estos valores nacen de los valores de pérdida de humedad reportados en la Tabla 1. Como se observa en las Figuras, la velocidad de pérdida de humedad de ambos alimentos disminuye con el incremento del tiempo y temperatura de freído, siendo mayores las pérdidas al inicio del proceso para la tilapia. Nasiri et al., (2011) indican que la pérdida de humedad es un importante fenómeno de transporte de masa durante la fritura, y que la tasa de este proceso está directamente relacionada con el tiempo de fritura y la temperatura, y Tirado et al., (2012) dice que esta disminución en la pérdida de humedad durante los primeros minutos se debe al aumento en el espesor de la corteza superficial, que trae consigo la disminución de la velocidad de transferencia de vapor en la superficie. 


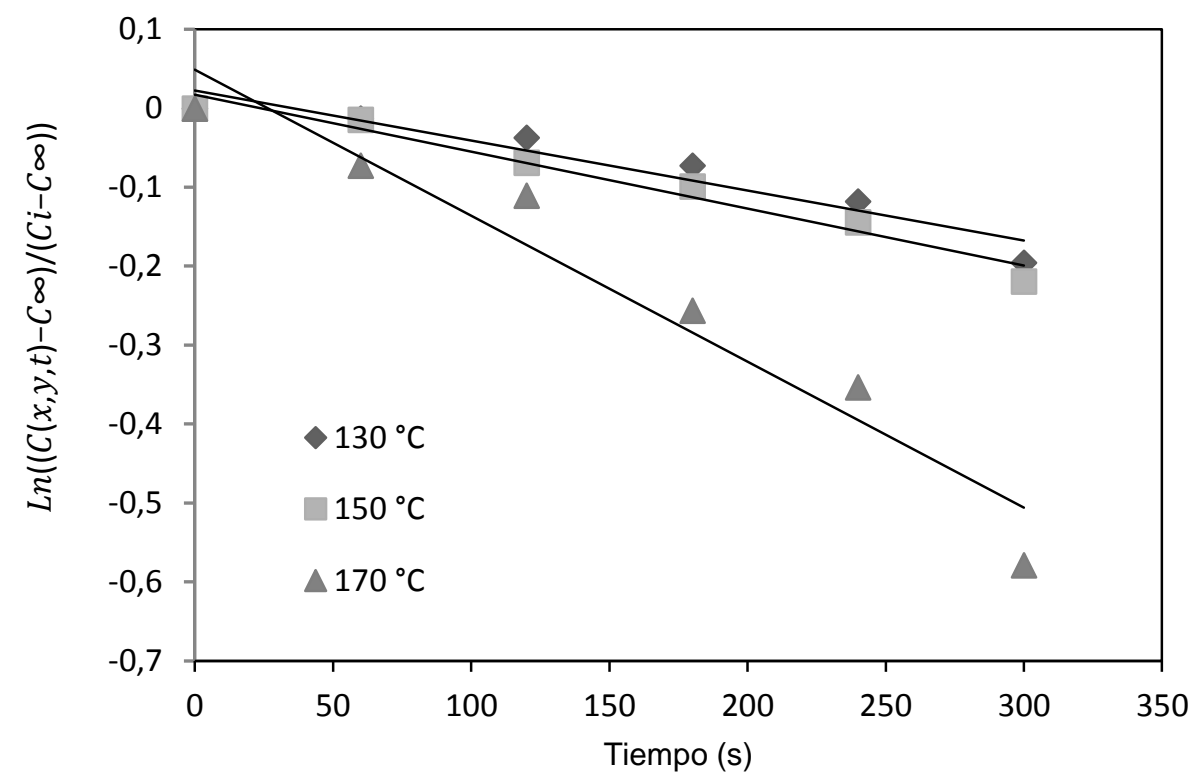

Fig. 1: Sección lineal de la relación adimensional humedad vs el tiempo en tilapia

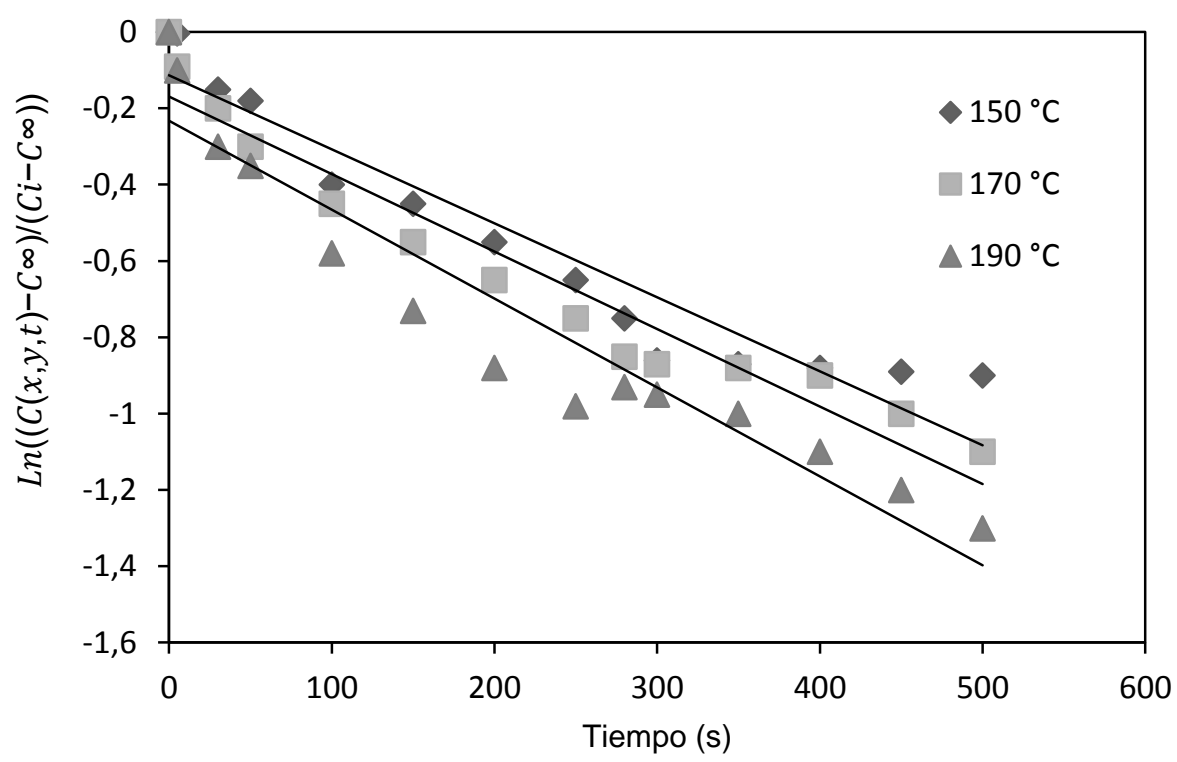

Fig. 2: Sección lineal de la relación adimensional humedad vs el tiempo en fruta de pan

Las pendientes de las secciones lineales de estas curvas, se obtuvieron mediante análisis de regresión lineal y fueron usadas posteriormente para determinar el coeficiente de transferencia de masa efectivo. De los tramos de mayor pendiente de las curvas se tomaron las necesarias para determinar el coeficiente de difusión, $\mathrm{D}_{\mathrm{a}}\left(\mathrm{m}^{2} / \mathrm{s}\right)$. Una vez hallado $\delta_{\mathrm{n}}$ se determinaron los valores del número de Biot, y posteriormente se realizaron los cálculos para los coeficientes de transferencia de masa (Ecuaciones 10 y 11). La Tabla 3 muestra finalmente los valores obtenidos de coeficientes de transferencia de masa $(\mathrm{kc})$, difusividad de la humedad $\left(D_{a}\right)$ y los respectivos coeficientes de regresión lineal para ambos alimentos. En la Tabla 3 se pueden evidenciar las diferencias entre los coeficientes de un alimento y otro, siendo estos mayores en la fruta de pan, muy posiblemente debido al tamaño de poros presente en cada alimento (Tirado et al., 2013).

En trabajos anteriores Yildiz et al., (2007) en la fritura de trozos de papa encontró coeficientes de transferencia de masa de $1,12,1,58$ y $2,07^{*} 10^{-5} \mathrm{~m} / \mathrm{s}$ a temperaturas entre 150 y $190^{\circ} \mathrm{C}$ respectivamente, y Alvis et al., (2009b) en el freído de trozos de ñame reportó valores para estos coeficientes entre 0,786 y $3,312^{*} 10^{-6} \mathrm{~m} / \mathrm{s}$ a temperaturas entre 140 y $180^{\circ} \mathrm{C}$. Estos valores son muy parecidos a los resultados obtenidos por las láminas de tilapia, pero difieren un poco con los conseguidos en la fritura de fruta de pan. Según Alvis et al., (2009b) estas diferencias pueden ser debidas al método y producto utilizado en el 
estudio, en este caso los alimentos de estudio presentan dos tipos de diferentes matrices: la matriz almidonada de la fruta de pan y la matriz proteica de la tilapia.

Tabla 3: Parámetros de transferencia de masa en función de la temperatura del aceite.

\begin{tabular}{|c|c|c|c|}
\hline \multicolumn{4}{|c|}{ Tilapia } \\
\hline $\begin{array}{l}\text { Temperatura de } \\
\text { aceite }\left({ }^{\circ} \mathrm{C}\right)\end{array}$ & $\begin{array}{l}\text { Coeficientes de } \\
\text { regresión lineal }\end{array}$ & $\begin{array}{l}\text { Coeficiente de transferencia } \\
\text { de masa }(\mathrm{m} / \mathrm{s})\end{array}$ & $\begin{array}{l}\text { Coeficiente de } \\
\text { difusividad }\left(\mathrm{m}^{2} / \mathrm{s}\right)\end{array}$ \\
\hline 130 & 0,0006 & $3,31^{\star} 10^{-6}$ & $2,85^{\star} 10^{-7}$ \\
\hline 150 & 0,0007 & $3,78^{\star} 10^{-6}$ & $3,25^{\star} 10^{-7}$ \\
\hline 170 & 0,0018 & $9,68^{\star} 10^{-6}$ & $8,46^{\star} 10^{-7}$ \\
\hline \multicolumn{5}{|c|}{ Fruta de pan } & $1,08^{\star} 10^{-10}$ \\
\hline 150 & 0,0019 & $6,60^{\star} 10^{-9}$ & $9,57^{*} 10^{-10}$ \\
\hline 170 & 0,002 & $6,96^{\star} 10^{-9}$ & $10,86^{\star} 10^{-10}$ \\
\hline 190 & 0,0023 & $8,03^{\star} 10^{-9}$ & \\
\hline
\end{tabular}

Referente al coeficiente de difusividad de la humedad Sosa-Morales et al., (2006) en fritura de lonjas de carne de cerdo obtuvieron valores de $1,59^{*} 10^{-9} ; 9,15^{*} 10^{-9}$ y $30,2^{*} 10^{-9} \mathrm{~m}^{2} / \mathrm{s}$ a temperaturas de 90,100 y 110 ${ }^{\circ} \mathrm{C}$, y Nasiri et al., (2011) en fritura de camarón encontraron valores entre $2,05^{*} 10^{-8}$ y $5,71^{*} 10^{-8} \mathrm{~m}^{2} / \mathrm{s}$ a temperaturas entre 150 y $190^{\circ} \mathrm{C}$, los cuales son muy similares a los obtenidos para la fruta del pan en este trabajo, aunque difieran para la tilapia. Sin embargo los valores de difusividad de humedad para la tilapia son similares a los datos reportados por otros autores. Dincer y Yildiz (1996), para salchichas fritas a $180^{\circ} \mathrm{C}$ encontró una difusividad de $1,31^{*} 10^{-7} \mathrm{~m}^{2} / \mathrm{s}$. Yildiz et al., (2007) en el freído de trozos de papa, obtuvieron valores de difusividad de la humedad de $9,2^{*} 10^{-9}, 11^{*} 10^{-9}$ y $18,2^{*} 10^{-9} \mathrm{~m}^{2} / \mathrm{s}$ a temperaturas de 150,170 y $190^{\circ} \mathrm{C}$; y por otro lado Alvis et al., (2009b) a 140,160 y $180^{\circ} \mathrm{C}$ halló coeficientes de $1,95,2,59$ y 3,24 * $10^{-}$ ${ }^{9} \mathrm{~m}^{2} / \mathrm{s}$ respectivamente. Los datos encontrados para la tilapia concuerdan con los de estos tres autores.

\section{Coeficientes de transferencia de calor}

Los coeficientes de transferencia de calor convectivo se determinaron de las pendientes de la sección lineal de la relación adimensional tiempo vs. temperatura análogamente que con los coeficientes de transferencia de masa. La Tabla 4 muestra los valores calculados del coeficiente convectivo de transferencia de calor $(h)$ con sus correspondientes números de Biot y coeficientes de regresión lineal. En la Tabla 4 se observa que los coeficientes disminuyeron al aumentar la temperatura del aceite en la tilapia. Caso contrario se presentó para la fruta de pan. Este último comportamiento es el más usual, y ha sido reportado por otros autores (Alvis et al., 2009b; Yildiz et al., 2007; Farinu y Baik, 2007; Sosa-Morales et al., 2006; Budzaki y Seruga, 2005; Costa y Oliveira, 1999; Califano y Calvelo, 1991). Sin embargo un mismo comportamiento que el de la tilapia también fue reportado por otros autores (Tirado et al., 2013; Mir-Bel et al., 2012; Yildiz et al., 2007). Según estos, esto puede ser debido a que el movimiento de las burbujas durante la fritura aumenta la tasa de transferencia de calor, mientras los niveles máximos de las velocidades de pérdida de agua pueden obstaculizar la transferencia de calor, y disminuir el coeficiente de transferencia de calor (Costa y Oliveira, 1999).

Tabla 4: Coeficientes de regresión lineal, Números de Biot y coeficientes convectivos

\begin{tabular}{|c|c|c|c|}
\hline \multicolumn{4}{|c|}{ Tilapia } \\
\hline Temperatura de aceite $\left({ }^{\circ} \mathrm{C}\right)$ & Coeficientes de regresión lineal & Número de Biot (Bi) & $h\left(W / m^{2} K\right)$ \\
\hline 130 & 0,0032 & 1,07 & 82,47 \\
\hline 150 & 0,0024 & 0,79 & 60,63 \\
\hline 170 & 0,0023 & 0,76 \\
\hline \multicolumn{4}{|c|}{ Fruta de pan } \\
\hline 150 & 0,0102 & 0,0244 & 65,85 \\
\hline 170 & 0,0133 & 0,0291 & 87,55 \\
\hline 190 & 0,0154 & 0,1724 & 102,98 \\
\hline
\end{tabular}

Los valores de $h$ calculados para la tilapia, se encuentran dentro del rango de valores reportados por SosaMorales et al., (2006) que ensayaron con placas de carne de cerdo de $0,05^{\star} 0,04^{\star} 0,02 \mathrm{~m}$ en aceite de girasol a 90,100 y $110^{\circ} \mathrm{C}$, encontrando coeficientes de transferencia de calor de $74,38 \pm 18,57,193,45 \pm 10,87$ y $226,10 \pm 14,29 \mathrm{~W} / \mathrm{m}^{2} \mathrm{~K}$. En estudios anteriores Tirado et al., (2013), bajo las mismas condiciones 
experimentales de esta investigación determinaron los coeficientes de transferencia de calor mediante el análisis de parámetros concentrados en la fritura de láminas de tilapia, encontrando coeficientes de 46,76, 34,61 y $32,66 \mathrm{~W} / \mathrm{m}^{2} \mathrm{~K}$, a las temperaturas de 130,150 y $170^{\circ} \mathrm{C}$. Para la determinación de sus cálculos no se tubo en cuenta la resistencia interna a la transferencia de calor $(\mathrm{Bi}<0,1)$, por lo cual se presentan valores de coeficientes diferentes a los reportados en este trabajo, aunque se manifiesta el mismo fenómeno de disminución de coeficientes al aumentar la temperatura del aceite. Estos resultados no indican que el estudio de Tirado et al., (2013) esté mal realizado, ya que en su trabajo calcularon los coeficientes obteniendo el mismo comportamiento que este estudio, pero si evidencia la importancia de tener en cuenta la resistencia interna a la transferencia de calor, lo cual ha sido de importancia en el cálculo de coeficientes para otros autores (Alvis et al., 2009; Yildiz et al., 2007). Sin embargo el análisis de parámetros concentrados es seleccionado por su sencillez frente a otros modelos, como el de diferencias finitas utilizado en este estudio. Este sencillo enfoque ha sido utilizado con los mismos objetivos en otros estudios tales como en el de Moreira et al., (1995a) para fritura de carne de cerdo y Moreira et al., (1995a) en fritura de chips de tortilla. En ambos trabajos, al igual que en el de Tirado et al., (2013), el mecanismo de convección es el control de la transferencia de calor, pero no tiene en cuenta la resistencia interna a la transmisión de calor en el alimento, es decir supone números de Biot (Bi) menores a 0,1 (Geankoplis, 1993). Los autores no encontramos otras investigaciones de determinación de coeficientes de transferencia de calor en tilapia u otro pescado a partir de la solución analítica de la ecuación diferencial de conducción de calor en una dimensión en coordenadas cartesianas para una placa infinita de espesor 2L.

Los resultados de los coeficientes convectivos de transferencia de calor calculados en esta investigación para la fruta de pan tienen el mismo comportamiento de los obtenidos por Alvis et al., (2009b) y Sahin et al., (1999), quienes trabajaron con ñame y papa respectivamente, y reportaron un incremento en el coeficiente de transferencia de calor por convección con el aumento de la temperatura de freído. Los valores de $h$ calculados para la fruta de pan, se encuentra dentro del rango de otras investigaciones como las de Budzaki y Seruga (2005) que hallaron valores de $h$ para esferas de una mezcla de harina de trigo y papa al freírlas en aceite de soya a $190^{\circ} \mathrm{C}$ durante 5 a $120 \mathrm{~s}$. Estos autores muestran que los valores más altos del coeficiente de transferencia de calor se observan en la etapa de ebullición y los valores mínimos, en la etapa de velocidad decreciente.

\section{CONCLUSIONES}

La velocidad de pérdida de humedad de ambos alimentos disminuye con el incremento del tiempo y temperatura de freído, siendo mayores las pérdidas al inicio del proceso para la tilapia. Los coeficientes de transferencia de masa y de difusividad de humedad aumentaron con el incremento de la temperatura en la tilapia y la fruta de pan, siendo mayores para este último, muy posiblemente debido al tamaño de poros presente en cada alimento. Los coeficientes convectivos de transferencia de calor disminuyeron al aumentar la temperatura del aceite en la tilapia. Caso contrario se presentó para la fruta de pan. Este último comportamiento es el más usual, y ha sido reportado por otros autores. Sin embargo un mismo comportamiento que el de la tilapia también fue reportado por otros autores, quienes afirman que el fenómeno es debido a que el movimiento de las burbujas durante la fritura aumenta la tasa de transferencia de calor, mientras los niveles máximos de las velocidades de pérdida de agua pueden obstaculizar la transferencia de calor, y disminuir el coeficiente de transferencia de calor. Se evidencia la importancia de tener en cuenta las resistencias internas a la transferencia de calor durante la fritura de tilapia.

\section{REFERENCIAS}

Ahmad, A., K. Niranjan, y M. Gordon, Physico-chemical changes occurring in oil when atmospheric frying is combined with post-frying vacuum application, Food Chemistry: 136(2), 902-908 (2013).

Aladedunye, F. y R. Przybylski, Frying stability of high oleic sunflower oils as affected by composition of tocopherol isomers and linoleic acid content, Food Chemistry: 141(3), 2373-2378 (2013).

Alvis, A. y otros tres autores, Heat transfer coefficient during deep-fat frying, Food Control: 20(4), 321-325 (2009a).

Alvis, A., L. Cortés y M. Páez, Transferencia de Calor y Materia durante la Fritura de Trozos de Ñame (Dioscórea alata), Información Tecnológica: 20(1), 99-109 (2009b).

Budzaki, S. B. y Seruga, Determination of convective heat transfer coefficient during frying of potato dough, Journal Food Engineering: 66(3), 307-314 (2005). 
Califano, A.N. y A. Calvelo, Thermal conductivity of potato between 50 and $100^{\circ} \mathrm{C}$, Journal of Food Science: 56, 586-589 (1991).

Costa, R.M. y F.A. Oliveira, Modelling the kinetics of water loss during potato frying with a compartmental dynamic model, Journal of Food Engineering: 41(3-4), 177-185 (1999).

Dincer, I. y M. Yildiz, Modelling of thermal and moisture diffusions in cylindrically shaped sausages during frying, Journal of Food Engineering: 28, 35 -43 (1996).

Farinu, A. y O.D. Baik, Heat transfer coefficients during deep fat frying of sweet potato: Effects of product size and oil temperature, Food Research International: 40(8), 989-994 (2007).

Geankoplis, C.J.; Transport Process and Unit Operation. 3 $3^{a}$ Edition, Prentice-Hall PTR, Englewood Cliffs, New Jersey. pp. 345 (1993).

Hsu, C.L. y otros cuatro autores, Cytotoxic effects of geranyl flavonoid derivatives from the fruit of Artocarpus communisin SK-Hep-1 human hepatocellular carcinoma cells, Food Chemistry: 127(1), 127-134 (2011).

Lalam, S. y otros cuatro autores, Experimental study on transport mechanisms during deep fat frying of chicken nuggets, LWT-Food Science and Technology: 50(1), 110-119 (2013).

Mir-Bel, J., R. Oria y M. Salvador, Influence of temperature on heat transfer coefficient during moderate vacuum deep-fat frying, Journal of Food Engineering: 113(2), 167-176 (2012).

Moreira, R. G., J. Palau y X. Sun, Simultaneous heat and mass transfer during deep fat frying of tortilla chips, Journal of Food Process Engineering: 18(3), 307-320 (1995a).

Moreira, R. y otros tres autores, Thermal and physical properties of tortilla chips as a function of frying time, Journal of Food Processing and Preservation: 19(4), 175-189 (1995b).

Moreira, R.G., M.E. Castell-Perez y M.A. Barrufet; Deep- fat frying: Fundamentals and applications, An aspen publication, Gaithersburg, Maryland, p.p 75-177 (1999).

Nasiri, F.D. y otros tres autores, Kinetic modeling of mass transfer during deep fat frying of shrimp nugget prepared without a pre-frying step, Food and Bioproducts Processing: 89(3), 241-247 (2011).

Sahin, S., S.K. Sastry, y L. Bayindirli, Heat transfer during frying of potato slices, Journal of Food Engineering: 32(1), 19-24 (1999).

Sosa-Morales, M.E., R. Orzuna-Espiritu y J.F. Velez-Ruiz, Mass, thermal and quality aspects of deep-fat frying of pork meat, Journal of Food Engineering: 77(3), 731-738 (2006).

Tirado, D.F., D. Acevedo y L.E. Guzmán, Coeficientes convectivos de transferencia de calor durante el freído de láminas de tilapia "Oreochromis niloticus", Inf. Tecnol. 24(6), 41-46 (2013).

Tirado, D.F., D. Acevedo y L.E. Guzmán, Freído por inmersión de los alimentos, Revista Reciteia: 12(1), 6982 (2012).

Tirado, D.F., D. Acevedo y P. Puello, Determinación Computacional del Coeficiente de Transferencia de Calor y Propiedades Termofísicas de Alimentos, Información Tecnológica: 25(3), En prensa (2014).

$\mathrm{Wu}, \mathrm{H}$. y otros tres autores, Analysis and simulation of continuous food frying processes, Applied Thermal Engineering: 53(2), 332-339 (2013b).

Wu, H., T.G. Karayiannis y S.A. Tassou, A two-dimensional frying model for the investigation and optimisation of continuous industrial frying systems, Applied Thermal Engineering: 51(1-2), 926-936 (2013a).

Yildiz, A.T., K. Palazoglu y F. Erdogdu, Determination of heat and mass transfer parameters during frying of potato slices, Journal of Food Engineering: 79(1), 11-17 (2007). 
